\title{
Clinical Outcomes of Second-Line Chemotherapy after Progression on Nab-Paclitaxel Plus Gemcitabine in Patients with Metastatic Pancreatic Adenocarcinoma
}

\author{
Kyoungmin Lee, MD \\ Kyunghye Bang, MD' \\ Changhoon Yoo, MD, PhD' \\ Inhwan Hwang, MD ${ }^{1}$ \\ Jae Ho Jeong, MD, $P h D^{1}$ \\ Heung-Moon Chang, MD, $\mathrm{PhD}^{1}$ \\ Dongwook Oh, MD² \\ Tae Jun Song, MD, PhD2 \\ Do Hyun Park, MD, PhD2 \\ Sang Soo Lee, MD, PhD2 \\ Sung Koo Lee, MD, PhD2 \\ Myung-Hwan Kim, MD, PhD² \\ Jin-hong Park, MD, PhD ${ }^{3}$ \\ Kyu-pyo Kim, MD, PhD ${ }^{1}$ \\ Baek-Yeol Ryoo, MD, PhD'
}

Departments of ${ }^{1}$ Oncology, ${ }^{2}$ Gastroenterology, and ${ }^{3}$ Radiation Oncology, Asan Medical Center, University of Ulsan College of Medicine, Seoul, Korea

\footnotetext{
Correspondence: Baek-Yeol Ryoo, MD, PhD Department of Oncology, Asan Medical Center, University of Ulsan College of Medicine, 88 Olympic-ro 43-gil, Songpa-gu,

Seoul 05505, Korea

Tel: 82-2-3010-3211

Fax: 82-2-3010-6961

E-mail: ryooby@amc.seoul.kr

Co-correspondence: Changhoon Yoo, MD, PhD Department of Oncology, Asan Medical Center, University of Ulsan College of Medicine,

88 Olympic-ro 43-gil, Songpa-gu,

Seoul 05505, Korea

Tel: 82-2-3010-1727

Fax: 82-2-3010-6961

E-mail: yooc@amc.seoul.kr

Received April 8, 2019

Accepted July 7, 2019

Published Online July 9, 2019

*Kyoungmin Lee and Kyunghye Bang contributed equally to this work.
}

\section{Purpose}

Since the introduction of nab-paclitaxel plus gemcitabine (nab-P+GEM) as first-line (1L) treatment for metastatic pancreatic adenocarcinoma (mPDAC), optimal second-line (2L) chemotherapy after progression is unclear. We assessed clinical outcomes of $2 \mathrm{~L}$ chemotherapy for disease that progressed on $1 \mathrm{~L}$ nab-P+GEM.

\section{Materials and Methods}

Among the 203 patients previously treated with $1 \mathrm{~L}$ nab-P+GEM for mPDAC at Asan Medical Center, between February and December 2016, records of 120 patients receiving $2 \mathrm{~L}$ chemotherapy after progression on nab-P+GEM were retrospectively reviewed. The response rate and survival were evaluated along with analysis of prognostic factors.

\section{Results}

Fluoropyrimidine-oxaliplatin doublets (FOLFOX or XELOX) were used in 78 patients (65.0\%), fluoropyrimidine monotherapy in 37 (30.8\%), and liposomal irinotecan plus fluorouracil in two (1.7\%). The median progression-free survival (PFS) and overall survival (OS) were 3.29 months and 7.33 months from the start of $2 \mathrm{~L}$ therapy. Fluoropyrimidine-oxaliplatin regimens and fluoropyrimidine monotherapy did not yield significantly different median PFS (2.89 months vs. 3.81 months, $p=0.40$ ) or OS (7.04 months vs. 7.43 months, $p=0.86$ ). A high neutrophil-lymphocyte ratio (> 2.2) and a short time to progression with $1 \mathrm{~L}$ nab-P+GEM (<6.4 months) were independent prognostic factors of poor OS with $2 \mathrm{~L}$ therapy.

\section{Conclusion}

$2 \mathrm{~L}$ fluoropyrimidine-oxaliplatin doublets and fluoropyrimidine monotherapy after failure of $1 \mathrm{~L}$ nab-P+GEM had modest efficacy, with no differences in treatment outcomes between them. Further investigation is warranted for the optimal $2 \mathrm{~L}$ chemo-regimens and sequencing of systemic chemotherapy for patients with mPDAC.

\section{Key words}

Pancreatic neoplasms, Nab-paclitaxel, Gemcitabine, Oxaliplatin, Second-line 


\section{Introduction}

Over the past two decades, gemcitabine had been the only first-line (1L) chemotherapeutic agent for treatment of advanced pancreatic ductal adenocarcinoma (PDAC) that has proven clinical benefit and modest improvement in survival compared with fluorouracil (5-FU) [1]. Recently, combination therapies such as FOLFIRINOX (5-FU, folinic acid, irinotecan, and oxaliplatin) and nab-paclitaxel plus gemcitabine (nab-P+GEM) have conferred superior outcomes compared with gemcitabine monotherapy. They have become the latest $1 \mathrm{~L}$ treatments for metastatic PDAC (mPDAC) $[2,3]$. Nonetheless, the disease in most patients eventually progresses despite new 1L therapy, and the prognosis remains dismal. The median overall survival (OS) of patients with mPDAC is less than 1 year [4].

Improved efficacy of $1 \mathrm{~L}$ chemotherapy may, however, afford a greater chance for patients with $\mathrm{mPDAC}$ to receive subsequent second-line (2L) chemotherapy. Innovations in $1 \mathrm{~L}$ chemotherapy, with attendant changes in their efficacy and safety profiles, may increase the possibility of achieving benefit from subsequent 2L therapy. Establishment of optimal sequences of effective chemotherapy regimens is needed to improve the overall clinical outcomes of patients with mPDAC in the era of modern $1 \mathrm{~L}$ regimens.

Currently, nab-P+GEM is a standard, widely used 1L regimen for mPDAC $[4,5]$. For patients whose cancer progresses on $1 \mathrm{~L}$ nab-P+GEM, fluoropyrimidine-based regimens, including fluoropyrimidine monotherapy, combined fluoropyrimidine and oxaliplatin, and liposomal irinotecan (nal-IRI) plus 5-FU and leucovorin (LV), are recommended as $2 \mathrm{~L}$ therapy in multiple guidelines [6-8]. These recommendations, however, were based on the results of earlier prospective studies [9-11] that were conducted prior to the introduction of $1 \mathrm{~L}$ nab-P+GEM. Since then, 2L chemotherapy after progression on 1L nab-P+GEM has been rarely investigated.

We therefore retrospectively analyzed clinical outcomes of $2 \mathrm{~L}$ chemotherapy in patients with mPDAC that had progressed on 1L nab-P+GEM.

\section{Materials and Methods}

\section{Patients and materials}

Between February and December 2016, 203 patients were treated with 1L nab-P+GEM for recurrent or metastatic pancreatic cancer at Asan Medical Center, Seoul, Korea, as previously reported [12]. The treatment was continued until disease progression, unacceptable toxicity, or patient or physician preference. For the current analysis, we retrospectively reviewed 120 patients who received $2 \mathrm{~L}$ chemotherapy after disease progression on 1L nab-P+GEM. Data collected from the medical records included clinical characteristics at the time 2L treatment was begun, including age, Eastern Cooperative Oncology Group performance status, number of metastatic sites, sites of metastasis, and levels of carbohydrate antigen 19-9 and albumin. The neutrophil, lymphocyte, and platelet counts were used to calculate neutrophil-lymphocyte ratio (NLR) and platelet-lymphocyte ratio (PLR).

\section{Assessment of treatment and response}

The $2 \mathrm{~L}$ chemotherapy regimen for each patient was chosen at the discretion of the attending physicians. During $2 \mathrm{~L}$ therapy, computed tomography or magnetic resonance imaging was performed every 6 to 8 weeks, with additional imaging if disease progression was suspected or unexpected symptoms arose. Tumor response was graded according to the Response Evaluation Criteria in Solid Tumors ver. 1.1.

\section{Statistical analysis}

Qualitative or categorical variables are presented as frequency and proportion and were compared using the chisquare test or Fisher exact test, as appropriate. Continuous variables, presented as median with $95 \%$ confidence interval (CI) or range, were compared using the test. Overall response rate (ORR) was defined as the proportion of patients who had a partial or complete response to $2 \mathrm{~L}$ therapy. The disease control rate (DCR) was a composite of ORR and stable disease.

OS with 2L treatment (2L-OS) was defined as the time from the start of $2 \mathrm{~L}$ chemotherapy to death, while total OS was defined as the time from the start of 1L nab-P+GEM to death. Time to progression (TTP) on 1L treatment (1L-TTP) was defined as the interval between the start of the $1 \mathrm{~L} n a b-\mathrm{P}+$ GEM and the date disease progression was first noted. Progression-free survival (PFS) with 2L treatment (2L-PFS) was defined as the time from the start of $2 \mathrm{~L}$ chemotherapy to subsequent disease progression or death. Data were censored if the disease had not progressed on the last evaluation or if patients were still alive at the time of data analysis (October 31, 2018). Survival curves were estimated by the KaplanMeier method and compared using the log-rank test.

Univariate Cox proportional hazard analysis was performed to estimate the impact of each clinicopathologic variable on 2L-OS and 2L-PFS. For these analyses, continuous variables were converted into binary variables based on their median values. Multivariate Cox analysis was further conducted to assess the simultaneous impact of various factors. 
Table 1. Baseline characteristics of patients with metastatic pancreatic ductal adenocarcinoma

\begin{tabular}{|lc|}
\hline & No. $(\%)(\mathbf{n}=\mathbf{1 2 0})$ \\
\hline Sex & \\
\hline Male & $70(58.3)$ \\
\hline Female & $50(41.7)$ \\
\hline Age, median (range, yr) & $61(35-79)$ \\
\hline ECOG performance status & \\
\hline 0 & $44(36.7)$ \\
\hline 1 & $74(61.7)$ \\
\hline 2 & $2(1.7)$ \\
\hline No. of metastatic sites & \\
\hline 0 & $4(3.3)$ \\
\hline 1 & $59(49.2)$ \\
\hline 2 & $34(28.3)$ \\
\hline 3 & $16(13.3)$ \\
\hline 4 & $7(5.8)$ \\
\hline Site of metastasis & \\
\hline Liver & $71(59.2)$ \\
\hline Lung & $30(25.0)$ \\
\hline Bone & $7(5.8)$ \\
\hline Peritoneum & $46(38.3)$ \\
\hline Lymph nodes & $40(33.3)$ \\
\hline 1L-TTP, median $\mathbf{9 5 \%}$ CI, mo) & $6.87(0.49-22.45)$ \\
\hline 2L chemotherapy regimens & \\
\hline FOLFOX & $56(46.7)$ \\
\hline XELOX & $22(18.3)$ \\
\hline S-1 & $37(30.8)$ \\
\hline Nal-IRI+5-FU/LV & $2(1.7)$ \\
\hline Investigational drugs & $3(2.5)$ \\
\hline
\end{tabular}

ECOG, Eastern Cooperative Oncology Group; 1L, first line; 2L, second line; TTP, time to progression; FOLFOX, folinic acid, fluorouracil, and oxaliplatin; XELOX, capecitabine plus oxaliplatin, S-1, tegafur/gimeracil/oteracil; Nal-IRI+5-FU/LV, liposomal irinotecan plus fluorouracil and leucovorin.

Using variables with a potential significant association by univariate analysis $(\mathrm{p}<0.2)$ as well as age and gender, backward elimination was applied to identify the best independent explanatory factors. All statistical analyses were performed using IBM SPSS Statistics for Windows ver. 21.0 (IBM Corp., Armonk, NY, USA). A value of $\mathrm{p}<0.05$ was considered statistically significant.

\section{Ethical statement}

This study was approved by the Institutional Review Board of Asan Medical Center (approval number: 2017-0442). Informed consent was waived because of the retrospective design.

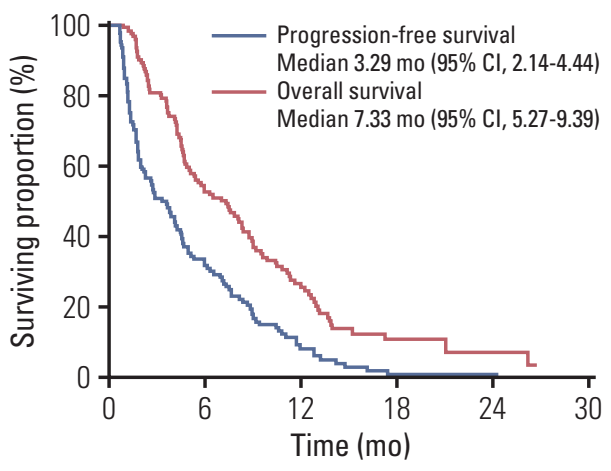

Fig. 1. Kaplan-Meier analyses of second-line progressionfree survival (blue line) and overall survival (red line) in all 120 patients. CI, confidence interval.

\section{Results}

\section{Patients and treatment regimens}

Among 203 patients who were treated with $1 \mathrm{~L}$ nab-P+GEM for recurrent or metastatic pancreatic cancer, 120 patients $(59.1 \%)$ received 2L chemotherapy after disease progression on $1 \mathrm{~L} n a b-\mathrm{P}+\mathrm{GEM}$ at Asan Medical Center, Seoul, Korea. Among other 83 patients, 43 and three patients did not have data on $2 \mathrm{~L}$ chemotherapy because they were transferred to other institutions or lost to follow-up during or after progression on $1 \mathrm{~L}$ nab-P+GEM, and were able to maintain primary treatment because the disease did not progress until the cutoff date, respectively; and, 25 and 12 patients did not receive $2 \mathrm{~L}$ chemotherapy because of poor general condition and patients' refusal, respectively. The median duration of $1 \mathrm{~L}$ treatment with nab-P+GEM was 6.87 months $(95 \% \mathrm{CI}, 6.19$ to 7.55). The median age of the patients was 61 years (range, 45 to 76 years), and 70 (58.3\%) were men. Most patients had a good performance status of 0 to 1 with the exception of two patients whose score was 2 . Most $(97.5 \%)$ received fluoropyrimidine-containing regimens as $2 \mathrm{~L}$ chemotherapy, except for three patients who participated in clinical trials of investigational drugs (Table 1). Regimens were as follows: FOLFOX (58 patients, $46.7 \%$ ): intravenous oxaliplatin $85 \mathrm{mg} / \mathrm{m}^{2}$, $\mathrm{LV} 400 \mathrm{mg} / \mathrm{m}^{2}$, and a 5 -FU bolus $400 \mathrm{mg} / \mathrm{m}^{2}$ on day 1 , followed by a $2,400 \mathrm{mg} / \mathrm{m}^{2}$ continuous 5 -FU infusion over 46 hours, every 2 weeks; XELOX (22 patients, 18.3\%): intravenous oxaliplatin $130 \mathrm{mg} / \mathrm{m}^{2}$ on day 1 and oral capecitabine $100 \mathrm{mg} / \mathrm{m}^{2}$ twice daily on days 1-14 every 3 weeks; Nal-IRI combined with 5-FU/LV (2 patients, 1.7\%): intravenous nalIRI $70 \mathrm{mg} / \mathrm{m}^{2}$ and LV $400 \mathrm{mg} / \mathrm{m}^{2}$, followed by a $2,400 \mathrm{mg} /$ $\mathrm{m}^{2}$ continuous infusion of 5 -FU over 46 hours, every 2 weeks; fluoropyrimidine monotherapy with S-1 (37 patients, 30.8\%): 
Table 2. Treatment outcomes with second-line chemotherapy

\begin{tabular}{|lcc} 
& $\begin{array}{c}\text { Fluoropyrimidine- } \\
\text { oxaliplatin doublets }(\mathbf{n = 7 8 )}\end{array}$ & $\begin{array}{c}\text { Fluoropyrimidine } \\
\text { monotherapy (n=37) }\end{array}$ \\
\hline Response, $\mathbf{n}(\%)$ & $5(6.4)$ & $2(5.4)$ \\
$\quad$ p-value
\end{tabular}

ORR, overall response rate; CR, complete response; CI, confidence interval; DCR, disease control rate; 2L-PFS, progressionfree survival for second-line treatment; 2L-OS, overall survival for second-line treatment.

A

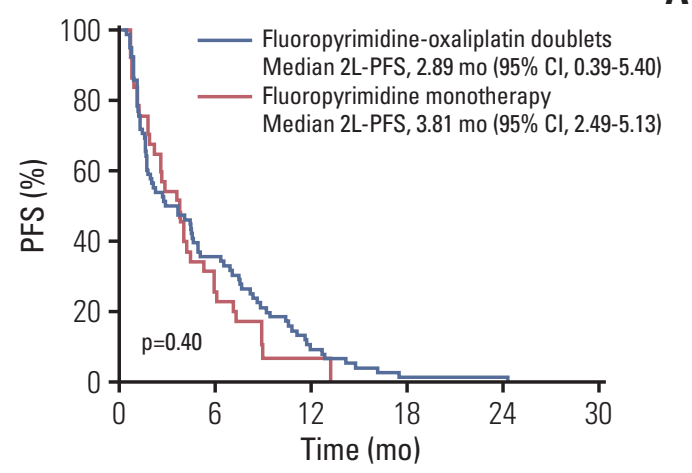

B

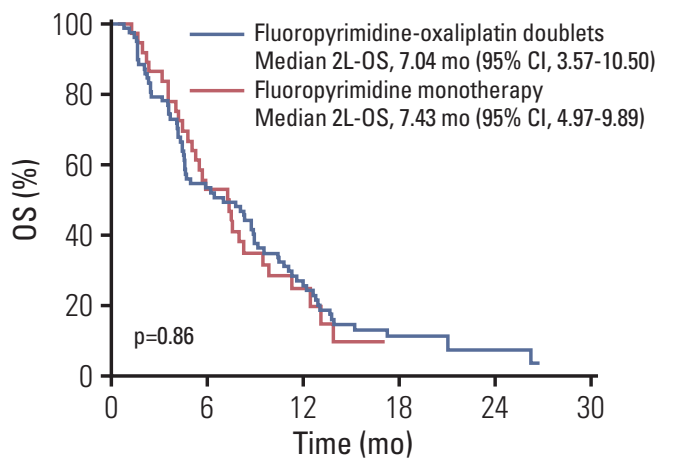

Fig. 2. Progression-free survival (PFS) (A) and overall survival (OS) (B) with second-line chemotherapy after progression on nab-paclitaxel plus gemcitabine (nab-P+GEM). 2L, second-line; CI, confidence interval.

oral S-1 $40 \mathrm{mg} / \mathrm{m}^{2}$ twice daily on days 1-28 every 6 weeks [13]. FOLFIRINOX was not used as 2L chemotherapy because it was not approved for use in Korea during the study period.

\section{Efficacy}

None of the regimens afforded a complete response. There were eight patients with a partial response and 57 with stable disease, indicating an ORR of $6.7 \%$ (95\% CI, 2.9 to 12.7) and a DCR of $54.2 \%$ (95\% CI, 44.8 to 63.3). With a median followup of 26.27 months (range, 3.28 to 31.92 months) after starting 1L chemotherapy, 115 patients had disease progression after 2L therapy, and 101 died. The median 2L-PFS and 2L-OS among all 120 patients were 3.29 months (95\% CI, 2.14 to 4.44) and 7.33 months (95\% CI, 5.27 to 9.39), respectively (Fig. 1). The median OS after the start of the 1L nab-P+GEM

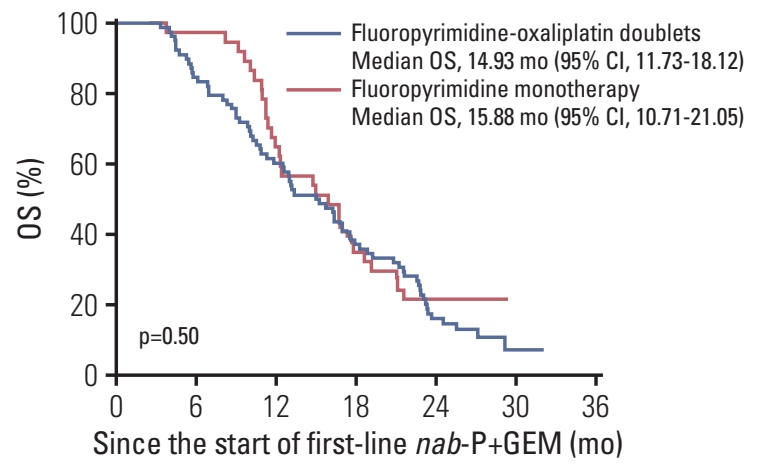

Fig. 3. Overall survival (OS) from the start of first-line nabpaclitaxel plus gemcitabine ( $n a b-\mathrm{P}+\mathrm{GEM}$ ) according to the second-line chemotherapy regimen. $\mathrm{CI}$, confidence interval. 
Table 3. Univariate and multivariate analysis of overall survival with second-line chemotherapy

\begin{tabular}{|c|c|c|c|c|}
\hline \multirow{3}{*}{ Sex } & \multicolumn{2}{|c|}{ Univariate analysis } & \multicolumn{2}{|c|}{ Multivariate analysis } \\
\hline & HR $(95 \%$ CI $)$ & p-value & \multirow[t]{2}{*}{ HR $(95 \%$ CI) } & \multirow[t]{2}{*}{ p-value } \\
\hline & & & & \\
\hline Male & Reference & & - & - \\
\hline Female & $0.94(0.63-1.41)$ & 0.77 & - & - \\
\hline \multicolumn{5}{|c|}{ Initial disease status } \\
\hline Recurrent & Reference & & - & - \\
\hline Metastatic & $1.09(0.70-1.69)$ & 0.70 & - & - \\
\hline \multicolumn{5}{|l|}{ Age (yr) } \\
\hline$\leq 60$ & Reference & & - & - \\
\hline$>60$ & $0.88(0.59-1.31)$ & 0.52 & - & - \\
\hline \multicolumn{5}{|c|}{ ECOG performance status } \\
\hline 0 & Reference & & - & - \\
\hline $1-2$ & $1.46(0.96-2.20)$ & 0.08 & - & - \\
\hline \multicolumn{5}{|l|}{ CA19-9 } \\
\hline Normal & Reference & & - & - \\
\hline Elevated & $1.12(0.63-1.99)$ & 0.70 & - & - \\
\hline \multicolumn{5}{|l|}{ Albumin } \\
\hline Normal & Reference & & - & - \\
\hline Decreased & $1.16(0.78-1.73)$ & 0.46 & - & - \\
\hline \multicolumn{5}{|l|}{ NLR } \\
\hline$\leq 2.2$ & Reference & & Reference & \\
\hline$>2.2$ & $1.78(1.19-2.66)$ & $<0.01$ & $1.58(1.05-2.38)$ & 0.03 \\
\hline \multicolumn{5}{|l|}{ PLR } \\
\hline$\leq 154.4$ & Reference & & - & - \\
\hline$>154.4$ & $1.57(1.05-2.36)$ & 0.03 & - & - \\
\hline \multicolumn{5}{|c|}{ No. of metastatic sites } \\
\hline $0-1$ & Reference & & Reference & \\
\hline$\geq 2$ & $1.60(1.07-2.38)$ & 0.02 & $1.48(0.99-2.22)$ & 0.06 \\
\hline \multicolumn{5}{|c|}{ Presence of liver metastasis } \\
\hline No & Reference & & - & - \\
\hline Yes & $1.77(1.16-2.69)$ & $<0.01$ & - & - \\
\hline \multicolumn{5}{|c|}{ Presence of lung metastasis } \\
\hline No & Reference & & - & - \\
\hline Yes & $0.97(0.62-1.52)$ & 0.88 & - & - \\
\hline \multicolumn{5}{|c|}{ Presence of bone metastasis } \\
\hline No & Reference & & - & - \\
\hline Yes & $2.05(0.943-4.44)$ & 0.07 & - & - \\
\hline \multicolumn{5}{|c|}{ Presence of peritoneal metastasis } \\
\hline No & Reference & & - & - \\
\hline Yes & $0.99(0.66-1.49)$ & 0.95 & - & - \\
\hline \multicolumn{5}{|c|}{ Presence of lymph nodes metastasis } \\
\hline No & Reference & & - & - \\
\hline Yes & $1.15(0.76-1.73)$ & 0.51 & - & - \\
\hline \multicolumn{5}{|l|}{ 1L-TTP (mo) } \\
\hline$\leq 6.4$ & $1.68(1.13-2.52)$ & 0.01 & $1.57(1.05-2.36)$ & 0.03 \\
\hline$>6.4$ & Reference & & Reference & \\
\hline \multicolumn{5}{|c|}{ Best response for first-line $n a b-\mathrm{P}+\mathrm{GEM}$} \\
\hline $\mathrm{CR} / \mathrm{PR} / \mathrm{SD}$ & Reference & & - & - \\
\hline PD & $1.38(0.83-2.31)$ & 0.22 & - & - \\
\hline
\end{tabular}

(Continued to the next page) 
Table 3. Continued

\begin{tabular}{|c|c|c|c|c|}
\hline & \multicolumn{2}{|c|}{ Univariate analysis } & \multicolumn{2}{|c|}{ Multivariate analysis } \\
\hline & HR $(95 \%$ CI $)$ & p-value & HR $(95 \% \mathrm{CI})$ & p-value \\
\hline \multicolumn{5}{|l|}{ Second-line regimen } \\
\hline Fluoropyrimidine monotherapy & Reference & & - & - \\
\hline Fluoropyrimidine-oxaliplatin doublets & $0.96(0.62-1.50)$ & 0.86 & - & - \\
\hline
\end{tabular}

HR, hazard ratio; CI, confidence interval; ECOG, Eastern Cooperative Oncology Group; CA19-9, carbohydrate antigen 19-9; NLR, neutrophil-lymphocyte ratio; PLR, platelet-lymphocyte ratio; $1 \mathrm{~L}$, first line; TTP, time to progression; nab-P+GEM, nab-paclitaxel plus gemcitabine; CR, complete response; PR, partial response; SD, stable disease; PD, progressive disease.

was 15.68 months (95\% CI, 12.53 to 18.83 ).

When the outcomes were compared among the 2L chemotherapy regimens, there were no significant differences in efficacy between fluoropyrimidine-oxaliplatin doublet therapy and fluoropyrimidine monotherapy in terms of 2L-PFS (median, 2.89 months [95\% CI, 0.39 to 5.40] vs. 3.81 months [95\% CI, 2.49 to 5.13]; hazard ratio [HR], 0.83 [95\% CI, 0.54 to 1.28]; $\mathrm{p}=0.40$ ), 2L-OS (median, 7.04 months [95\% CI, 3.57 to 10.50] vs. 7.43 months [95\% CI, 4.97 to 9.89]; HR, 0.96 [95\% CI, 0.62 to 1.50$] ; p=0.86)$, ORR (6.4\% vs. $5.4 \%, p>0.99)$, and DCR (52.6\% vs. 59.5\%, p=0.49) (Table 2, Fig. 2). The median OS from the start of 1L nab-P+GEM was 14.93 months (95\% $\mathrm{CI}, 11.73$ to 18.12 ) for patients receiving fluoropyrimidineoxaliplatin combination treatments and 15.88 months (95\% $\mathrm{CI}, 10.71$ to 21.05 ) for patients receiving fluoropyrimidine monotherapy. There was no significant between-group differences in terms of total OS (HR, 1.16 [95\% CI, 0.75 to 1.79]; $\mathrm{p}=0.50)$ (Fig. 3).

\section{Third-line chemotherapy}

Among 115 patients with progression on 2L chemotherapy, $53(46.1 \%)$ subsequently had third-line (3L) chemotherapy. There was no significant difference in the frequency of $3 \mathrm{~L}$ treatment between patients whose disease progressed on 2L fluoropyrimidine-oxaliplatin doublets $(38 / 77,49.4 \%)$ versus those on fluoropyrimidine monotherapy $(14 / 34,41.2 \%)$ $(\mathrm{p}=0.43)$. After progression on 2L fluoropyrimidine-oxaliplatin doublet regimens, fluoropyrimidine monotherapy was the most frequently used 3L chemotherapy $(31 / 38,81.6 \%)$, followed by nal-IRI plus 5-FU/LV (4/38, 10.5\%). Most patients whose disease progressed on fluoropyrimidine monotherapy received a 3L oxaliplatin doublet regimen (12/14, $85.7 \%)$, and two patients $(2 / 14,14.3 \%)$ received nal-IRI plus 5-FU / LV (S1 Table).

\section{Prognostic factors for survival with $2 \mathrm{~L}$ chemotherapy}

On univariate analysis, factors significantly associated with a poor 2L-OS included a high NLR (> median [2.2]), high PLR ( $>$ median [154.4]), $\geq 2$ organs with metastases, presence of liver metastasis, and a short 1L-TTP $(\leq$ median [6.4 months]). On multivariate analysis, high NLR (> 2.2; HR, $1.58 ; 95 \% \mathrm{CI}, 1.05$ to $2.39 ; \mathrm{p}=0.03)$ and a short $1 \mathrm{~L}-\mathrm{TTP}(\leq 6.4$; $\mathrm{HR}, 1.57 ; 95 \% \mathrm{CI}, 1.05$ to 2.36 ; $\mathrm{p}=0.03$ ) were independent predictors of a poor 2L-OS2 (Table 3). For 2L-PFS, the presence of liver metastasis (HR, 1.51; 95\% CI, 1.03 to 2.23; $\mathrm{p}=0.04$ and adjusted $\mathrm{HR}, 1.51 ; 95 \% \mathrm{CI}, 1.02$ to $2.24 ; \mathrm{p}=0.04$ ) was the only statistically significant factor on both univariate and multivariate analyses (S2 Table). There was no difference in these prognostic factors between patients receiving fluoropyrimidine-oxaliplatin doublets and those treated with fluoropyrimidine monotherapy (S3 Table).

\section{Discussion}

This study showed that $59.1 \%$ of patients with mPDAC treated with $1 \mathrm{~L}$ nab-P+GEM subsequently received $2 \mathrm{~L}$ chemotherapy, most commonly fluoropyrimidine-based chemotherapy. The median 2L-PFS and 2L-OS with 2L therapy after progression on 1L nab-P+GEM were 3.29 months and 7.33 months, respectively. Our findings are consistent with the results of post-hoc analysis for patients enrolled in the MPACT trial, in which $40 \%$ of patients assigned to the nab-P+GEM arm subsequently had $2 \mathrm{~L}$ therapy resulting in a median $\mathrm{OS}$ of 5.3 months (95\% CI, 4.17 to 5.78) [14]. Our results are also comparable in terms of total OS from the start of 1L nab-P+ GEM. The median was 15.68 months (95\% CI, 12.53 to 18.83 ) in our cohort and 12.8 months $(95 \% \mathrm{CI}, 10.9$ to 14.2$)$ in the MPACT post-hoc analysis [14]. Our survival outcomes with $2 \mathrm{~L}$ chemotherapy are in line with the results of previous phase III trials for 2L chemotherapy (PFS, 1.5 to 3.1 months; OS, 3.3 to 9.9 months) after failure of gemcitabine-based chemotherapy conducted prior to the establishment of the 1L nab-P+GEM regimen [9-11]. 
Although fluoropyrimidine-based chemotherapy is recommended as $2 \mathrm{~L}$ therapy after progression on $1 \mathrm{~L}$ nab-P+GEM, this continues to be debated. At present, FOLFIRINOX, fluoropyrimidine-oxaliplatin doublets such as FOLFOX or oxaliplatin plus LV and 5-FU (OFF), nal-IRI plus 5-FU/LV, and fluoropyrimidine monotherapy such as 5-FU/LV, capecitabine, or S-1 are available as 2L chemotherapy after failure of 1 L gemcitabine-based therapy. S-1, an oral fluoropyrimidine, is approved for the treatment of advanced PDAC in East Asia based on the non-inferiority of S-1 compared with gemcitabine monotherapy as $1 \mathrm{~L}$ therapy in the randomized phase III GEST trial [15]. Fluoropyrimidine-oxaliplatin regimens have been widely used since the OFF regimen (oxaliplatin 85 $\mathrm{mg} / \mathrm{m}^{2}$ before LV $200 \mathrm{mg} / \mathrm{m}^{2}$, followed by a continuous infusion of 5 -FU $2,000 \mathrm{mg} / \mathrm{m}^{2}$ over 24 hours on days 8 and 22) showed a significantly better OS than 5-FU/LV (LV 200 $\mathrm{mg} / \mathrm{m}^{2}$ followed by a continuous infusion of 5 -FU 2,000 $\mathrm{mg} / \mathrm{m}^{2}$ over 24 hours on days $1,8,15$, and 22) in the phase III CONKO-003 trial [9]. However, Japanese phase II trial comparing S-1 plus oxaliplatin with S-1 alone and the recent phase III PANCREOX study with a more commonly used biweekly administration schedule (mFOLFOX6: oxaliplatin $85 \mathrm{mg} / \mathrm{m}^{2}$ plus LV $400 \mathrm{mg} / \mathrm{m}^{2}$ and $400 \mathrm{mg} / \mathrm{m}^{2}$ of bolus FU followed by $2,400 \mathrm{mg} / \mathrm{m}^{2}$ of 5 -FU continuous infusion for 46 hours, every 14 days vs. biweekly LV $400 \mathrm{mg} / \mathrm{m}^{2}$ and a 400 $\mathrm{mg} / \mathrm{m}^{2}$ of bolus FU followed by $2,400 \mathrm{mg} / \mathrm{m}^{2}$ of 5 -FU continuous infusion for 46 hours, every 14 days) failed to show a clinical benefit with the addition of oxaliplatin to fluoropyrimidine $[10,16]$. The nal-IRI plus $5-\mathrm{FU} / \mathrm{LV}$ regimen is the newest option for patients with mPDAC that progresses on $1 \mathrm{~L}$ gemcitabine, as indicated by its success in the NAPOLI-1 trial [11].

In the current study, there was no significant difference in efficacy between fluoropyrimidine-oxaliplatin doublets and fluoropyrimidine monotherapy for $2 \mathrm{~L}$ salvage treatment. This finding is in line with the results of the Japanese Phase II trial [16] and PANCREOX trial [10], but in contrast to those of the CONKO-003 trial [9]. Discrepancies in the efficacy of oxaliplatin reported among the studies may be attributed to the heterogeneous baseline characteristics of the different patient populations as well as differences in study design or dosing schedules. Oxaliplatin-containing chemotherapy may have limitations as $2 \mathrm{~L}$ therapy after progression on $1 \mathrm{~L}$ nab-P+ GEM in terms of tolerability considering that a substantial proportion of patients treated with nab-P+GEM suffer from neuropathy. Although oxaliplatin-containing triplet chemotherapy (i.e., FOLFIRINOX) as 2L chemotherapy may have better clinical outcomes in medically fit patients with mPD$\mathrm{AC}$, we could not evaluate that question in our study, as no patient in the study cohort was treated with 2L FOLFIRINOX. Considering the relatively high risk of toxicities with FOLFIRINOX, it is unlikely to be universally accepted as $2 \mathrm{~L}$ therapy after $1 \mathrm{~L}$ nab-P+GEM. Further studies are needed to elucidate the clinical relevance of $2 \mathrm{~L}$ oxaliplatin-containing chemotherapy following $1 \mathrm{~L}$ nab-P+Gem for mPDAC.

Irinotecan-fluoropyrimidine doublets have also been investigated in patients with mPDAC [17]. While combining fluoropyrimidine with conventional irinotecan did not show a clear clinical benefit, nal-IRI plus 5-FU/LV significantly improved survival compared with 5-FU/LV, and this regimen received regulatory approval. Recent real-world data showed consistent clinical outcomes with nal-IRI plus 5-FU/ $\mathrm{LV}$ in patients with $\mathrm{mPDAC}$ who had previously received gemcitabine-based chemotherapy $[18,19]$. Because of the heterogeneity of $1 \mathrm{~L}$ chemotherapy prior to the administration of nal-IRI plus 5-FU/LV and different treatment lines (from 1st- to 5th-line) when it was used in those studies, however, it is difficult to estimate the efficacy of 2L nal-IRI plus 5-FU/ LV after 1L nab-P+GEM. We also could not evaluate its efficacy due to the small number of patients in our study who had been treated with 2L nal-IRI plus 5-FU/LV.

Of note, the median survival in our cohort of more than 15 months after starting $1 \mathrm{~L}$ therapy suggests that effective $2 \mathrm{~L}$ chemotherapy after 1L nab-P+GEM may lead to a relatively long-term survival in patients with mPDAC. Our analysis showed that $59.1 \%$ of patients with $1 \mathrm{~L}$ nab-P+GEM could receive $2 \mathrm{~L}$ therapy and subsequent $3 \mathrm{~L}$ chemotherapy was given in $49.1 \%$ of $2 \mathrm{~L}$ patient population. Although our analysis is limited to discuss the overall outcomes of $1 \mathrm{~L}$ nab-P+ GEM, because we included only 2L population, current findings may indicate that subsequent $2 \mathrm{~L}$ and $3 \mathrm{~L}$ chemotherapy may prolong the survival of patients with mPDAC who started 1L nab-P+GEM. Although there is no head-to-head comparative trial, FOLFIRINOX showed numerically better survival outcomes compared to nab-P+GEM in 1L setting $[2,3]$, recent retrospective analyses and meta-analysis in realworld setting have revealed that survival outcomes with these regimens may be similar in mPDAC patients $[4,20]$. The efficacy of subsequent 2L and 3L chemotherapy after progression on 1L nab-P+GEM shown in our study may contribute to these results. However, it is still difficult to clearly demonstrate the contribution of $2 \mathrm{~L}$ chemotherapy after nab$\mathrm{P}+\mathrm{GEM}$ with the current data alone. Considerable work is needed to fully understand the implications of subsequent chemotherapy and to determine appropriate salvage therapy strategy.

The NLR at the start of 2L chemotherapy was shown to be predictive of 2L-OS in our cohort. Consistent with our results, although the underlying mechanism is not yet known, a high NLR has been suggested as a poor prognostic factor for patients with pancreatic cancer [21-23]. Multivariate post-hoc analysis of the MPACT trial revealed that an NLR $\leq 5$ at the end of $1 \mathrm{~L}$ gemcitabine-based therapy was associated with longer post-1L survival [14], which is also compatible with 
our findings. Moreover, a long 1L-PFS ( $\geq 4.4$ months) in that analysis was also associated with longer survival. This is consistent with our finding that a short TTP after $1 \mathrm{~L}$ nab-P+GEM therapy ( $\leq 6.4$ months) was associated with poor 2L-OS.

This study is limited by being a single-center study and by its retrospective design. Another limitation is the heterogeneity of the $2 \mathrm{~L}$ regimens used. These were chosen by attending physicians based not only individual patients' clinical factors but also on reimbursement policies in Korea. However, the fact that all patients in the study had received the same, new $1 \mathrm{~L}$ regimen made our cohort more homogenous, and this is the first real-world study evaluating outcomes of salvage treatment for mPDAC previously treated with nab-P+GEM.

In conclusion, 2L fluoropyrimidine monotherapy and fluoropyrimidine-oxaliplatin doublets after failure of $1 \mathrm{~L}$ nab-
P+GEM had modest efficacy. There was no difference in efficacy outcomes between fluoropyrimidine-oxaliplatin doublet chemotherapy and fluoropyrimidine monotherapy. Further investigation is warranted for the optimal $2 \mathrm{~L}$ chemotherapy regimens and sequencing of systemic chemotherapy for patients with mPDAC in multicenter prospective trials.

\section{Electronic Supplementary Material}

Supplementary materials are available at Cancer Research and Treatment website (https: // www.e-crt.org).

\section{Conflicts of Interest}

Conflict of interest relevant to this article was not reported.

\section{References}

1. Burris HA 3rd, Moore MJ, Andersen J, Green MR, Rothenberg ML, Modiano MR, et al. Improvements in survival and clinical benefit with gemcitabine as first-line therapy for patients with advanced pancreas cancer: a randomized trial. J Clin Oncol. 1997;15:2403-13.

2. Conroy T, Desseigne F, Ychou M, Bouche O, Guimbaud R, Becouarn $\mathrm{Y}$, et al. FOLFIRINOX versus gemcitabine for metastatic pancreatic cancer. N Engl J Med. 2011;364:1817-25.

3. Von Hoff DD, Ervin T, Arena FP, Chiorean EG, Infante J, Moore $\mathrm{M}$, et al. Increased survival in pancreatic cancer with nab-paclitaxel plus gemcitabine. N Engl J Med. 2013;369:1691703.

4. Kang J, Hwang I, Yoo C, Kim KP, Jeong JH, Chang HM, et al. $\mathrm{Nab}$-paclitaxel plus gemcitabine versus FOLFIRINOX as the first-line chemotherapy for patients with metastatic pancreatic cancer: retrospective analysis. Invest New Drugs. 2018;36:73241.

5. Abrams TA, Meyer G, Meyerhardt JA, Wolpin BM, Schrag D, Fuchs CS. Patterns of chemotherapy use in a U.S.-based cohort of patients with metastatic pancreatic cancer. Oncologist. 2017;22:925-33.

6. National Comprehensive Cancer Network. NCCN clinical practice guidelines in oncology (NCCN Guidelines $®)$ ). Pancreatic adenocarcinoma. Version 1.2019 [Internet]. Plymouth Meeting, PA: National Comprehensive Cancer Network; 2019 [cited 2019 Feb 4]. Available from: https://www.nccn.org/ professionals / physician_gls / pdf / pancreatic.pdf.

7. Ducreux M, Cuhna AS, Caramella C, Hollebecque A, Burtin P, Goere D, et al. Cancer of the pancreas: ESMO Clinical Practice Guidelines for diagnosis, treatment and follow-up. Ann Oncol. 2015;26 Suppl 5:v56-68.

8. ESMO Guidelines Committee. Appendix 6: cancer of the pancreas: MCBS eUpdate published online 20 June 2017 (www.
esmo.org/Guidelines / Gastrointestinal-Cancers). Ann Oncol. 2017;28(suppl_4):iv157.

9. Oettle H, Riess H, Stieler JM, Heil G, Schwaner I, Seraphin J, et al. Second-line oxaliplatin, folinic acid, and fluorouracil versus folinic acid and fluorouracil alone for gemcitabine-refractory pancreatic cancer: outcomes from the CONKO-003 trial. J Clin Oncol. 2014;32:2423-9.

10. Gill S, Ko YJ, Cripps C, Beaudoin A, Dhesy-Thind S, Zulfiqar $\mathrm{M}$, et al. PANCREOX: a randomized phase III study of fluorouracil/ leucovorin with or without oxaliplatin for secondline advanced pancreatic cancer in patients who have received gemcitabine-based chemotherapy. J Clin Oncol. 2016;34:391420.

11. Wang-Gillam A, Li CP, Bodoky G, Dean A, Shan YS, Jameson $\mathrm{G}$, et al. Nanoliposomal irinotecan with fluorouracil and folinic acid in metastatic pancreatic cancer after previous gemcitabine-based therapy (NAPOLI-1): a global, randomised, open-label, phase 3 trial. Lancet. 2016;387:545-57.

12. Hwang I, Kang J, Ip HN, Jeong JH, Kim KP, Chang HM, et al. Prognostic factors in patients with metastatic or recurrent pancreatic cancer treated with first-line nab-paclitaxel plus gemcitabine: implication of inflammation-based scores. Invest New Drugs. 2019;37:584-90.

13. Morizane C, Okusaka T, Furuse J, Ishii H, Ueno H, Ikeda M, et al. A phase II study of S-1 in gemcitabine-refractory metastatic pancreatic cancer. Cancer Chemother Pharmacol. 2009;63: 313-9.

14. Chiorean EG, Von Hoff DD, Tabernero J, El-Maraghi R, Ma WW, Reni M, et al. Second-line therapy after nab-paclitaxel plus gemcitabine or after gemcitabine for patients with metastatic pancreatic cancer. Br J Cancer. 2016;115:188-94.

15. Ueno H, Ioka T, Ikeda M, Ohkawa S, Yanagimoto H, Boku N, et al. Randomized phase III study of gemcitabine plus S-1, 
S-1 alone, or gemcitabine alone in patients with locally advanced and metastatic pancreatic cancer in Japan and Taiwan: GEST study. J Clin Oncol. 2013;31:1640-8.

16. Ohkawa S, Okusaka T, Isayama H, Fukutomi A, Yamaguchi K, Ikeda M, et al. Randomised phase II trial of S-1 plus oxaliplatin vs S-1 in patients with gemcitabine-refractory pancreatic cancer. Br J Cancer. 2015;112:1428-34.

17. Yoo C, Hwang JY, Kim JE, Kim TW, Lee JS, Park DH, et al. A randomised phase II study of modified FOLFIRI.3 vs modified FOLFOX as second-line therapy in patients with gemcitabinerefractory advanced pancreatic cancer. Br J Cancer. 2009;101: 1658-63.

18. Glassman DC, Palmaira RL, Covington CM, Desai AM, Ku GY, Li J, et al. Nanoliposomal irinotecan with fluorouracil for the treatment of advanced pancreatic cancer, a single institution experience. BMC Cancer. 2018;18:693.

19. Yoo C, Im HS, Kim KP, Oh DY, Lee KH, Chon HJ, et al. Multicenter retrospective analysis for efficacy and safety of liposomal irinotecan (nal-IRI) plus 5-FU/leucovorin (5-FU/LV) after progression on gemcitabine-based therapy in Korean patients (pts) with metastatic pancreatic ductal adenocarcinoma (mPDAC): a study by Korean Cancer Study Group (KCSG). J Clin Oncol. 2019;37(4 Suppl):Abstr 344.

20. Pusceddu S, Ghidini M, Torchio M, Corti F, Tomasello G, Niger M, et al. Comparative effectiveness of gemcitabine plus Nab-Paclitaxel and FOLFIRINOX in the first-line setting of metastatic pancreatic cancer: a systematic review and metaanalysis. Cancers (Basel). 2019;11:E484.

21. Sugiura T, Okamura Y, Ito T, Yamamoto Y, Ashida R, Yoshida $\mathrm{Y}$, et al. Prognostic scoring system for patients who present with a gastric outlet obstruction caused by advanced pancreatic adenocarcinoma. World J Surg. 2017;41:2619-24.

22. Chen Y, Yan H, Wang Y, Shi Y, Dai G. Significance of baseline and change in neutrophil-to-lymphocyte ratio in predicting prognosis: a retrospective analysis in advanced pancreatic ductal adenocarcinoma. Sci Rep. 2017;7:753.

23. Yang JJ, Hu ZG, Shi WX, Deng T, He SQ Yuan SG. Prognostic significance of neutrophil to lymphocyte ratio in pancreatic cancer: a meta-analysis. World J Gastroenterol. 2015;21:280715. 